\title{
Evaluation of deformations of thin-walled pipes during cold bending with rolling
}

\author{
A. V. Kozlov ${ }^{1}$, E. V. Khaliulin ${ }^{1}$, S. I. Platov' ${ }^{2}$ M. V. Nalimova ${ }^{\dagger, 2}$, V. A. Nekit ${ }^{2}$ \\ †lite59@mail.ru \\ ${ }^{1}$ South Ural State University, 76 Lenin Av., Chelyabinsk, 454080, Russia \\ ${ }^{2}$ Nosov Magnitogorsk State Technical University, 38 Lenin Av., Magnitogorsk, 455000, Russia
}

\begin{abstract}
The results of experimental evaluation of deformations of thin-walled pipes during their cold bending with rolling are discussed in this paper. The review of application of curvilinear elements of pipelines is considered. The methodology of the experiment is presented. Special equipment for cold bending pipes with rolling was used for experiments. The grid method was used as the basis for the strain estimation method. The measurements were carried out with an electronic caliper and a microscope of the MIM-340 model with an accuracy of $0.01 \mathrm{~mm}$. The evaluation of deformations for various materials and pipe diameters is given in detail: steel Ck10, steel X10CrNiTi18-9 and brass 2.0280. The values of elongation and reduction of the length of the walls of the pipe involved in the beating were determined. For a general assessment of the deformations and residual stresses, summary comparative graphs of changes in the geometric shapes of pipe samples after bending with rolling were constructed by the method of studying the absolute elongations of pipe walls during bending with rolling. It is established that the degree of deformation of pipes obeys a certain regularity. Of a particular interest is the bending of pipes made of corrosion-resistant steel. In this regard, similar experiments were carried out on bending of the pipes from corrosionresistant steel X10CrNiTi18-9. The thickness of the pipe walls and its length are calculated for three different variants of the pipe material, and their average range is given. As a result of the studies, dependencies were obtained that can be used to determine the nature of elongation and reduce the length of the pipe walls at various places of curved pipes, to determine the degree and nature of deformation in the longitudinal and cross sections of the pipe walls during bending with rolling and, as a result, to calculate these deformations and thinning of the pipe walls, which is an important operational characteristic.
\end{abstract}

Keywords: pipes, bending, rolling, deformation, stress.

УДК: 621.774

\section{Оценка деформаций тонкостенных труб при холодной гибке с раскатыванием}

\author{
Козлов А. В. ${ }^{1}$, Халиулин Е. В. ${ }^{1}$, Платов С. И. ${ }^{2}$, Налимова М. В. ${ }^{\dagger}, 2$, Некит В. А. ${ }^{2}$ \\ ${ }^{1}$ Южно-Уральский государственный университет, пр. Ленина, 76, Челябинск, 454080, Россия \\ ${ }^{2}$ Магнитогорский государственный технический университет им. Г.И. Носова, пр. Ленина, 38, \\ Магнитогорск, 455000, Россия
}

В статье рассмотрены результаты экспериментальной оценки деформаций тонкостенных труб при их холодной гибке с раскатыванием. Показана область применения криволинейных элементов трубопроводов. Приведена методика проведения экспериментов. При проведении экспериментов использовалось специальное оборудование для холодной гибки труб с раскатыванием. В основе способа оценки деформаций труб был использован метод сеток. Для измерения использовались электронный штангенциркуль и микроскоп модели МИМ-340 с точностью измерения до 0.01 мм. Подробно приведена оценка деформаций для различных материалов и диаметров труб: стали 10, стали 12X18Н9Т и латуни Л68. Для общей оценки деформаций и остаточных напряжений методом исследования абсолютных удлинений стенок трубы при гибке с раскатыванием построены сводные сравнительные графики изменений геометрических форм образцов труб после гибки с раскатыванием. Особый интерес представляет гибка труб из коррозионностойких сталей. В связи с этим были проведены аналогичные эксперименты по гибке труб из коррозионностойких сталей марки 12Х18Н9Т. Рассчитаны толщины стенок трубы и ее длина для трех 
различных материалов трубы. В результате проведённых исследований были получены зависимости, по которым можно установить характер удлинения и уменьшения длины стенок трубы в различных местах изогнутых труб, удалось оценить степень и характер деформации в продольном и поперечном сечениях стенок трубы при гибке с раскатыванием и, как следствие, рассчитать указанные деформации и утонения стенок трубы, что является важной эксплуатационной характеристикой.

Ключевые слова: трубы, гибка, раскатывание, деформации, напряжения.

\section{1. Введение}

Гибка труб является одной из основных операций технологического процесса изготовления деталей трубопроводов, которые находят широкое применение в авиации, машиностроении, атомной энергетике, нефтегазовой промышленности и др. отраслях [1-8].

В ЮУрГУ была разработана новая технология холодной гибки труб с раскатыванием (Рис. 1). Сущность технологии заключается в том, что при вращении раскатника, заведенного в трубу с достаточно большим натягом, в каждой точке кольцевой зоны раскатывания возникает знакопеременный изгиб, при котором изгибные напряжения кратковременно достигают предела текучести $\sigma_{\text {т. }}$ В результате при приложении относительно небольшого изгибающего усилия происходит гибка трубы в перемещающейся кольцевой зоне раскатывания (где $\omega_{1}-$ скорость подачи изгибаемой трубы; $\omega_{2}-$ скорость вращения раскатника).

При этом, по сравнению с известными методами гибки труб, изменяется характер деформаций и в продольном, и в поперечном сечениях: происходит удлинение внешней стенки трубы, укорачивание внутренней стенки, изменяется длина боковой стенки, что влияет на срок службы трубы [9-12].

Целью исследования является определение и оценка деформаций тонкостенных труб при холодной гибке с раскатыванием.

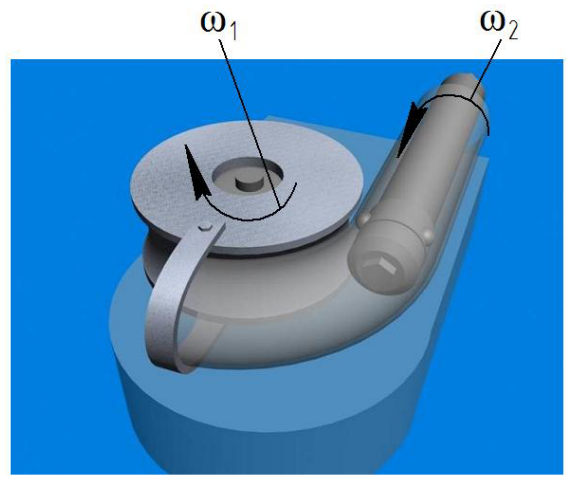

\section{2. Методика проведения исследования}

Для экспериментальной оценки степени деформации стенок трубы была проведена гибка труб разных диаметров из разных материалов на специальном оборудовании для холодной гибки труб с раскатыванием [6,13-16]. Данные об исследуемых образцах труб занесены в Табл. 1. Схема разметки и измерения интервалов приведена на Рис. 2.

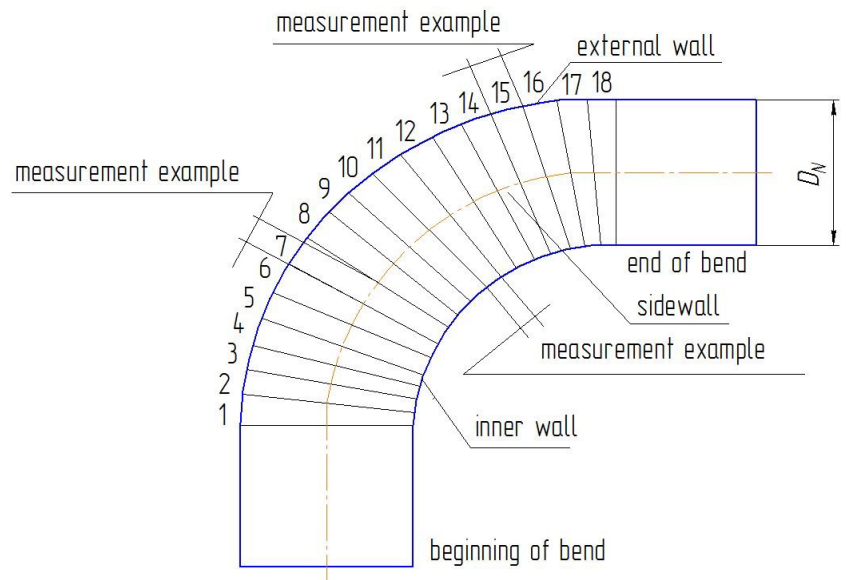

Рис. 2. Схема разметки и измерения интервалов, участвующих в гибке.

Fig. 2. The layout and measurement of the intervals involved in bending.

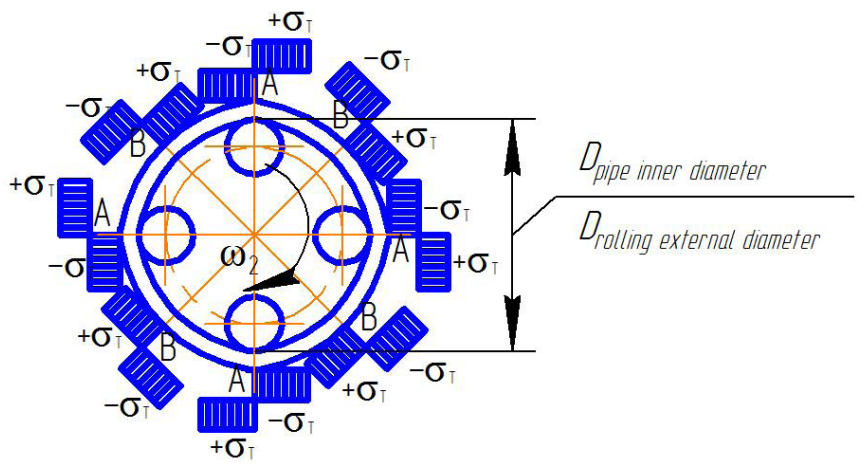

Pис. 1. (Color online) Схема гибки труб, раскатываемых с большими натягами.

Fig. 1. (Color online) The scheme of bending pipes which are rolled out with large tightness.

Табл. 1. Образцы труб для гибки.

Table 1. Samples of pipes for bending.

\begin{tabular}{|c|c|c|c|c|}
\hline № & $\begin{array}{c}\text { Наружный диаметр, мм } \\
\text { Outer diameter, mm }\end{array}$ & $\begin{array}{c}\text { Внутренний диаметр, мм } \\
\text { Internal diameter, mm }\end{array}$ & $\begin{array}{c}\text { Угол гиба } \\
\text { Bend angle }\end{array}$ & Материал / Material \\
\hline 1 & 60 & 54 & 90 & Сталь 10/ Steel Ck10 \\
\hline 2 & 26.5 & 21.5 & 90 & Сталь 10/ Steel Ck10 \\
\hline 3 & 26.5 & 21.5 & 90 & Латунь Л68 / Brass 2.0280 \\
\hline 4 & 42.5 & 40.25 & 90 & Сталь 12Х18H9T / Steel X10CrNiTi18-9 \\
\hline
\end{tabular}




\section{3. Оценка деформаций}

Для оценки деформаций тонкостенных труб был использован метод сеток [13]. Каждая труба перед гибкой была разградуирована кольцевыми рисками через каждые 5 мм. После изгиба труб проводились измерения расстояний между рисками с использованием инструментального микроскопа модели МИМ-340 (Рис. 3) $[13,15,17]$.

Для оценки деформаций были построены графические зависимости изменений геометрических форм образцов труб после гибки: график $1-$ труба $D=60$, сталь 10 ; график $2-$ труба $D=26.5$, сталь 10 ; график $3-$ труба $D=26.5$, латунь Л68 (Рис. $4-6$ ).

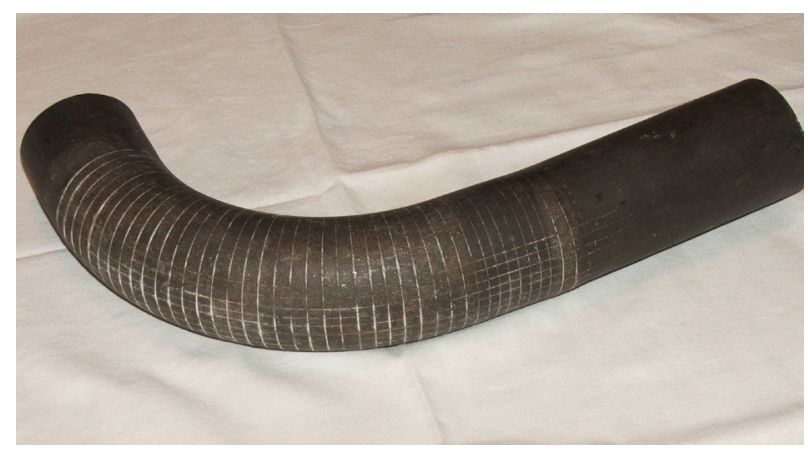

Рис. 3. Образец размеченной трубы: $D=60$ мм, материал: сталь 10 . Fig. 3. Sample of marked pipe: $D=60 \mathrm{~mm}$, material: steel Ck10.

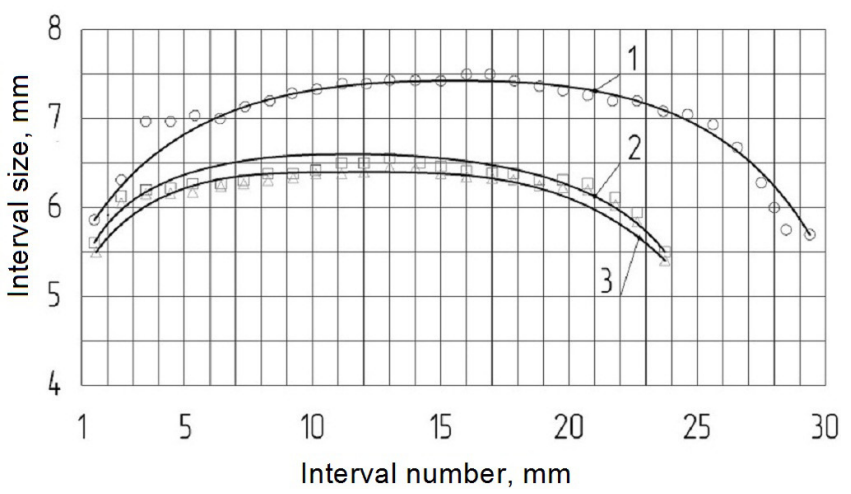

Рис. 4. График изменений абсолютных величин длин интервалов в местах гиба по внешним стенкам труб.

Fig. 4. Graph of changes in the absolute values of the lengths of the intervals in places of bending along the outer walls of pipes.

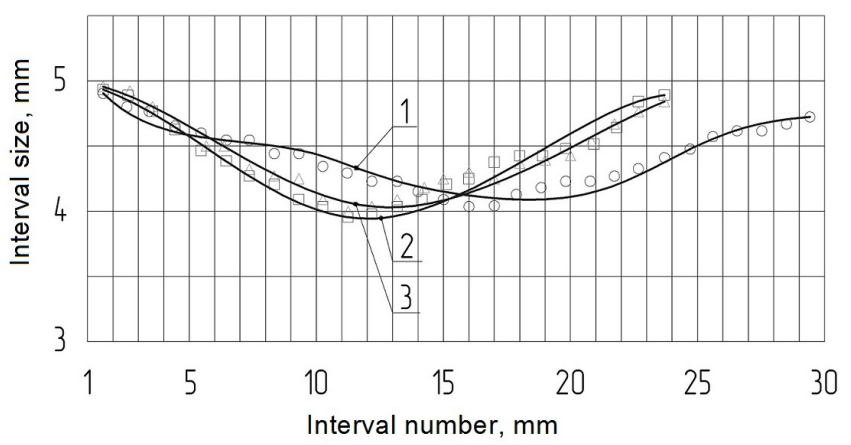

Рис. 5. График изменений абсолютных величин длин интервалов в местах гиба по внутренним стенкам труб.

Fig. 5. Graph of changes in the absolute values of the lengths of the intervals in the places of bending along the inner walls of the pipes.

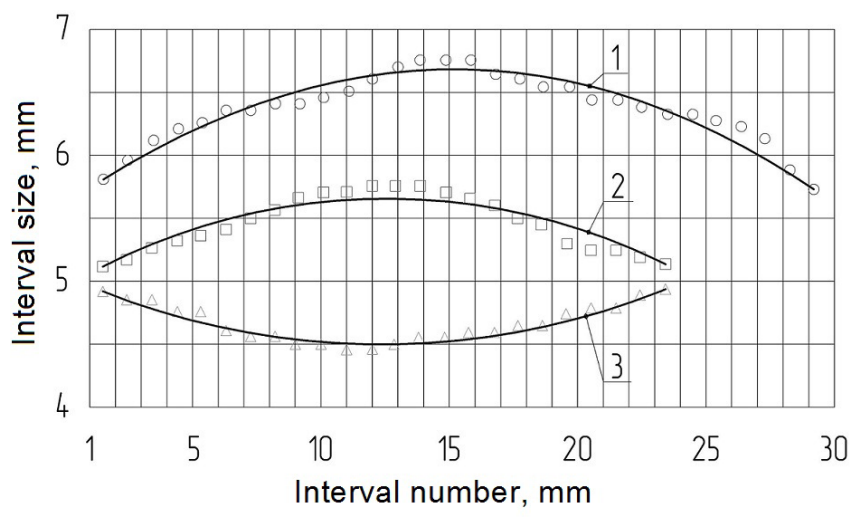

Рис. 6. График изменений абсолютных величин длин интервалов в местах гиба по боковым стенкам труб.

Fig. 6. Graph of changes in the absolute values of the lengths of the intervals in the places of bending along the inner walls of the pipes.

\section{4. Сравнение полученных зависимостей}

Из полученных зависимостей видно, что степень деформации труб изменяется по определенной закономерности в зависимости от конкретного материала. Из графиков видны определенные отличия, связанные с различными материалами изгибаемых труб. Для сравнения построим график зависимости изменений общих деформаций в месте гиба путем сведения всех полученных предыдущих графических зависимостей к общей закономерности (Рис. 7). От начала гиба продольные и поперечные деформации постепенно увеличиваются к середине гиба. В середине гиба абсолютные удлинения внешней, боковой и внутренней стенок труб различны, и, как следствие, гофрообразование в середине гиба является максимальным. К концу гиба абсолютные удлинения на внешней, боковой стенках постепенно уменьшаются [13,18].

Путем проведения исследования удалось оценить степень и характер деформации продольной и поперечной форм труб при гибке и, как следствие, оценить качественный характер распределения остаточных напряжений в образцах согнутых труб $[11,15,17]$.

Особый интерес представляет гибка труб из коррозионностойких сталей. В связи с этим были проведены аналогичные эксперименты по гибке труб из коррозионностойких сталей марки $12 \mathrm{X} 18 \mathrm{H} 9 \mathrm{~T}$.

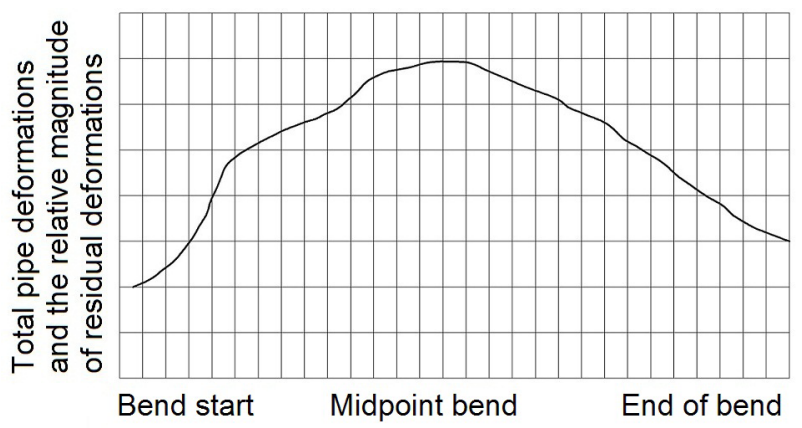

Рис. 7. График зависимости изменений качественного характера распределения внутренних напряжений.

Fig. 7. Graph of dependence of changes of qualitative character of distribution of internal tension. 
В результате были получены графические зависимости, по которым можно понять характер удлинения и уменьшения длины стенок трубы $[19,20]$ в различных местах гиба: 1 график - измерение по внутренней стенки трубы, 2 график - измерение по боковой стенки трубы, 3 график - измерение по наружной стенки трубы (Рис. 8). Анализируя полученные графики, можно установить определенную закономерность изменений деформаций на различных участках изгибаемой трубы при различных натягах (Табл. 2). Величина натяга, т.е. разница между диаметром раскатника и внутренним диаметром трубы, колебалась в пределах от 0.5 до 1.5 мм.

Анализ структуры металла после гибки с раскатыванием подтвердил отсутствие видимых повреждений. В то же время, имеет место уменьшение пластичности материала трубы. Так, относительное удлинение уменьшается в 2-3 раза, в то же время после отжига пластичность частично восстанавливается $[21,22]$.

По полученным деформациям можно впоследствии оценить толщину стенок на разных участках изогнутой трубы, что действительно является важной эксплуатационной характеристикой. Ранее нами были выполнены теоретические расчеты изменения толщин стенки изгибаемой трубы на различных участках.

Табл. 2. Изменения деформаций на различных участках изгибаемой трубы при различных натягах.

Table 2. Change of deformations in different parts of the bent pipe at different tightness.

\begin{tabular}{|c|c|c|c|}
\hline $\begin{array}{c}\text { Натяг, мм } \\
\text { Tightness, mm }\end{array}$ & $\begin{array}{c}\text { Точка 1 } \\
\text { Point 1 }\end{array}$ & $\begin{array}{c}\text { Точка 2 } \\
\text { Роint 2 }\end{array}$ & $\begin{array}{c}\text { Точка 3 } \\
\text { Роint 3 }\end{array}$ \\
\hline 0.5 & $-7.4 \%$ & $12.0 \%$ & $35.2 \%$ \\
\hline 1 & $-11.0 \%$ & $16.8 \%$ & $36.8 \%$ \\
\hline 1.5 & $-12.0 \%$ & $18.0 \%$ & $39.4 \%$ \\
\hline
\end{tabular}

\section{5. Заключение}

В результате проведенного исследования удалось оценить деформации в продольном и поперечном сечении стенок трубы, согласно построенным графическим зависимостям: на внутренней стенке наблюдалось уменьшение длины стенок трубы в среднем от 7.4\% до $12.0 \%$, на боковой стенке наблюдалось удлинение стенки трубы от $12.0 \%$ до $18.0 \%$, на наружной стенке наблюдалось удлинение стенки трубы в среднем от $35.2 \%$ до 39.4\%. Полученные результаты проведенных экспериментов позволяют прогнозировать величины утонения стенок трубы, а, следовательно, и общий срок службы: на внутренней стенки наблюдалось утолщение стенок трубы в среднем от $16.0 \%$ до $26.3 \%$, на боковой стенки наблюдалось утонение стенки трубы от $4.2 \%$ до $10.4 \%$, на внешней стенки наблюдалось утонение стенок трубы в среднем от $14.5 \%$ до $21.8 \%$.

\section{Литература/References}

1. Yu.V. Gorokhov, V.G. Sherkunov, N.N. Dovzhenko, S. V. Belyaev, I.N. Dovzhenko. Osnovy proyektirovaniya protsessov nepreryvnogo pressovaniya metallov. Monograph. Krasnoyarsk, Institute of non-ferrous metals and materials science, Siberian Federal University (2013) 224 p. (in Russian) [Ю.В. Горохов, В.Г. Шеркунов, Н. Н. Довженко, С. В. Беляев, И. Н. Довженко. Основы проектирования процессов непрерывного прессования металлов. Монография. Красноярск, Институт цветных металлов и материаловедения, Сибирский федеральный университет (2013) 224 с.]

2. M.Yu. Semashko, V.G. Sherkunov, P.A. Chigintsev. Vestnik of Nosov Magnitogorsk State Technical University. 1 (41), 57 (2013). (in Russian) [М.Ю. Семашко, В.Г. Шеркунов, П.А. Чигинцев. Вестник Магнитогорского государственного технического университета им. Г.И. Носова. 1 (41), 57 (2013).]

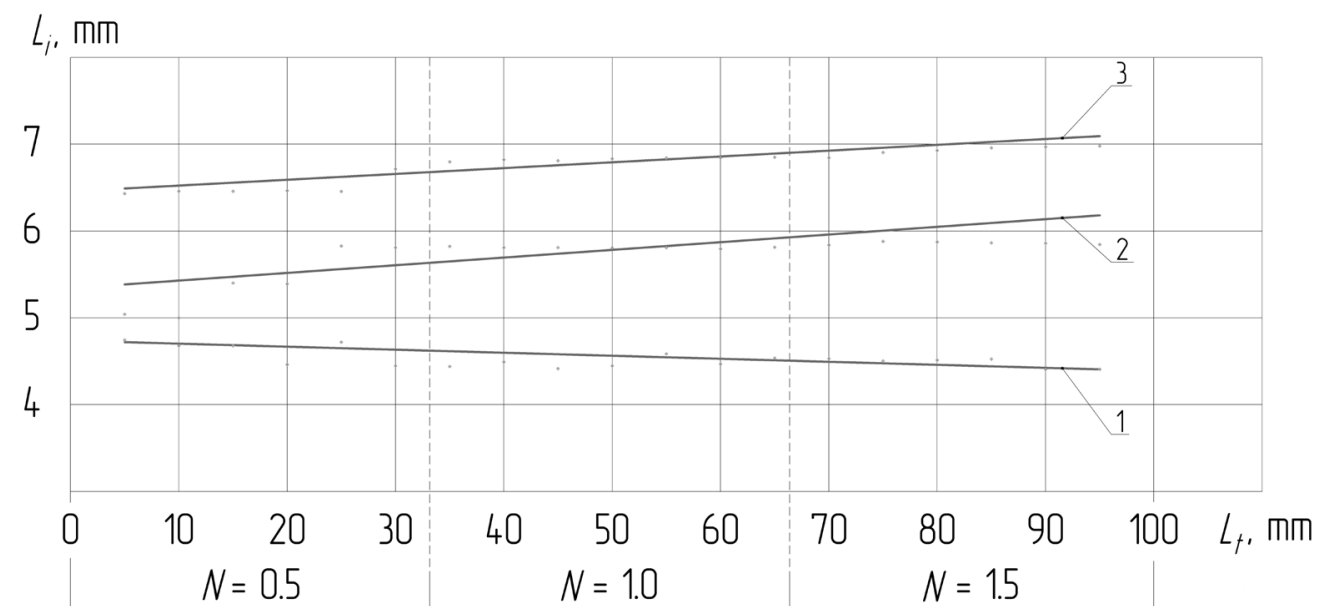

Рис. 8. Графики изменения интервалов, где $L_{i}$ - длина интервалов, $L_{t}-$ длина измеряемого участка трубы: зона (а) натяг $N=0.5$ мм; зона (b) натяг $N=1$ мм; зона (c) натяг $N=1.5$ мм.

Fig. 8. Graphs of change of the intervals, where $L_{i}$ is the length of the intervals, $L_{t}$ is the length of the measured pipe section: zone (a) of tightness $N=0.5 \mathrm{~mm}$; zone (b) tightness $N=1 \mathrm{~mm}$; zone (c) tightness $N=1.5 \mathrm{~mm}$. 
3. N.A. Fomenko, S.V. Aleksikov, S.G. Artyomova International Conference on Industrial Engineering, ICIE 2016. Procedia Engineering. Chelyabinsk (2016) p. 1359. Crossref

4. S. O. Gaponenko, A.E. Kondratiev, A.R. Zagretdinov. International Conference on Industrial Engineering, ICIE 2016. Procedia Engineering. Chelyabinsk (2016) p. 2321. Crossref

5. M. Herrmann, M. Fiderer, J. Walters. Papers of the International Conference "New Developments in Forging Technology" in Fellbach (near Stuttgart). MATINFO Werkstoff-Informationsgesellschaft. Frankfurt/M. (2005) 68.

6. N. V. Sudakov, V. I. Truskovsky, V. G. Sherkunov. Izvestiya Tula State University. Technical sciences. 6 (2), 98 (2013). (in Russian) [Н. В. Судаков, В.И. Трусковский, B. Г. Шеркунов. Известия Тульского государственного университета. Технические науки. 6 (2), 98 (2013).]

7. B. A. Lopatin, T. R. Khaziev. International Conference on Industrial Engineering, ICIE 2015. Procedia Engineering. Chelyabinsk (2015) p. 563. $\underline{\text { Crossref }}$

8. L. V. Plotnikov, B. P. Zhilkin, Yu. M. Brodov. International Conference on Industrial Engineering, ICIE 2016. Procedia Engineering. Chelyabinsk (2016) p. 111. Crossref

9. V. V. Shirokov, A. V. Vydrin. Stal'. 1, 30 (2016). (in Russian) [В. В. Широков, А. В. Выдрин. Сталь. 1, 30 (2016).]

10. Patent RF. № 818707/B. I. 13 (1981). (in Russian) [Патент РФ. № 818707/Б. И. 13 (1981).]

11. A. V. Kozlov, V.G. Sherkunov, Ya.M. Kyilcevic. Russian Engineering Reseach. 29 (8), 809 (2009). Crossref

12. Patent RF № 157963/B. I. 35 (2015). (in Russian) [Патент РФ. № 157963. У/Б. И. 35 (2015).]

13. N.M. Belyaev. Strength of materials. Moscow, Nauka (1976) 608 p. (in Russian) [Н. М. Беляев. Сопротивление материалов. Москва, Наука (1976) 608 с.]

14. A.I. Halperin. Pipe bending machines and equipment Moscow, Engineering (1983) 203 p. (in Russian)
[А. И. Гальперин. Машины и оборудование для гибки труб. Москва, Машиностроение (1983) 203 с.]

15. A. V. Kozlov, A. V. Bobylev. Tekhnologiya i oborudovaniye kholodnoy gibki tonkostennykh trub. Monograph. Chelyabinsk, Publishing house of SUSU (2007) 169 p. (in Russian) [А. В. Козлов, А. В. Бобылев. Технология и оборудование холодной гибки тонкостенных труб. монография. Челябинск. Изд-во ЮУрГУ (2007) 169 с.]

16. E. V. Khaliulin, A. V. Kozlov. Bulletin of the South Ural State University. Series «Engineering». 18 (3), 58 (2018). (in Russian) [Е.В. Халиулин, А.В.Козлов. Вестник ЮУрГУ. Серия «Машиностроение». 18 (3), 58 (2018).] Crossref

17. U.S. Johnson, P. B. Mellor. Plasticity Theory for Engineers. Moscow, Engineering (1979) 567 p. (in Russian) [У.С. Джонсон, П.Б. Меллор Теория пластичности для инженеров. Москва, Машиностроение (1979) 567 c.]

18. A.V. Vydrin, E. V. Khramkov, M.V. Bunyashin. Stahl und Eisen. 3, 47 (2017). (in Russian) [А. В. Выдрин, Е. В. Храмков, М. В. Буняшин. Черные металлы. 3, 47 (2017).

19. S.P. Minatsevich, A.A. Sharonov, S.S. Borisov. International Conference on Industrial Engineering, ICIE 2015. Procedia Engineering. Chelyabinsk (2015) p. 266. $\underline{\text { Crossref }}$

20. E. V. Moskvicheva, P.A. Sidyakin, D.V. Shitov. International Conference on Industrial Engineering, ICIE 2016. Procedia Engineering. Chelyabinsk (2016) p. 2381. Crossref

21. A. V. Kozlov, I. V. Chumanov, A. V. Bobylev. Izvestiya vuzov. Chernaya metallurgiya. 6, 33 (1999). (in Russian) [А. В. Козлов, И. В. Чуманов, А. В. Бобылев. Известия вузов. Черная металлургия. 6, 33 (1999).]

22. A.V. Kozlov, V.G. Sherkunov. Vestnik of Nosov Magnitogorsk State Technical University. 3, 32 (2009). (in Russian) [А. В. Козлов, В. Г. Шеркунов. Вестник МГТУ им. Носова. 3, 32 (2009).] 